J. Nepal Chem. Soc., Vol. 23, 2008/2009

\title{
Synthesis and Biological Activity of Novel 1-(Substituted Phenoxyacetoxy) Alkyl Phosphonates and Phosphinates
}

\author{
Liping Meng ${ }^{1,2}$, Rajendra Joshi ${ }^{3}$, Meiqiang $\mathrm{Li}^{1}$ and Hongwu $\mathrm{He}^{\mathbf{1}^{*}}$ \\ 1. Key Lab. of Pesticide \& Chemical Biology, Central China Normal University, Wuhan, P. R. China \\ 2. Department of Chemistry, University of California, Davis, CA 95616, USA \\ 3. Institute of Organic Chemistry, University of Tuebingen, 72076 Tuebingen, Germany \\ e-mail: he1208@mail.ccnu.edu.cn
}

\begin{abstract}
To investigate the influence of nitro group on the biological activity of phenoxyacetoxy phosphonates and phosphinates, a series of novel 1-(substituted phenoxyacetoxy) alkyl phosphonates and phosphinates were synthesized and screened for herbicidal activity and plant growth regulatory activity. Compounds $\mathbf{5 a}, \mathbf{5 b}$ and $\mathbf{5 j}$ exhibited notable bioactivity. Results indicated that nitro groups had a great influence on the biological activity, and the improvement of biological activity required a reasonable combination of a nitro group and other substituents.
\end{abstract}

\section{Introduction}

It is known that substituted phenoxyacetic acid derivatives possess herbicidal activity. ${ }^{1-3}$ Some acetylphosphinates and acetylphosphonates showed modest herbicidal activity due to their inhibition against pyruvate dehydrogenase complex $(\mathrm{PDHc}){ }^{4,5}$ In recent years, we have been engaged in designing agrochemicals utilizing a biochemically rational approach. ${ }^{6,7}$ The general structure (1) shown in Fig. 1, which possessed significant herbicidal activities, could act as a leading structure for herbicide design and some of them also showed plant growth regulatory activity.

Structure modification of (1) has been attempted by introducing different substituents R, X and Y to the phosphonates. ${ }^{8,9}$ However, none of our previous work was devoted towards examining the effects of nitro group substituents on herbicidal activity and plant growth regulatory activity. Here, we would like to introduce the $\mathrm{NO}_{2}$ group in $\mathrm{R}, \mathrm{NO}_{2}$ and $\mathrm{Cl}$ as $\mathrm{X}$ or/and $\mathrm{Y}$ into the phosphonates and their corresponding phosphinates to explore the effects on bioactivity. A novel series of 1-

\footnotetext{
* Corresponding author
} 
(substituted phenoxyacetoxy) alkyl phosphonates and phosphinates were synthesized and screened for herbicidal activity and plant growth regulatory activity.

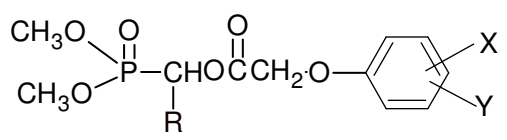

Figure 1: The general structure of O,O-dimethyl 1-(substituted phenoxyacetoxy) alkyl phosphonates.

\section{Experimental Methods}

Melting points were determined with an X4 melting-point apparatus and were uncorrected. Refractive index was measured with an Abbe refractometer at $20^{\circ} \mathrm{C}$. Elemental analyses were performed with a PE-2400 elemental analysis apparatus. IR spectra were obtained with a Perkin-Elmer-983 spectrometer. ${ }^{1} \mathrm{H}$ NMR spectra were recorded on a Varian XL-400 spectrometer with TMS as internal standard and $\mathrm{CDCl}_{3}$ as solvent. Column chromatography $(30 \times 3 \mathrm{~cm}$ column) was carried out using silica gel. 2,4-Dichlorophenoxy acetic acid was purchased from Yancheng Huilong Chemical Co. Ltd, China. All other chemicals and solvents used were of reagent grade. Compounds 1, 2, 3 and 4, as shown in Scheme 1, were prepared according to the literature. . $^{10-15}$

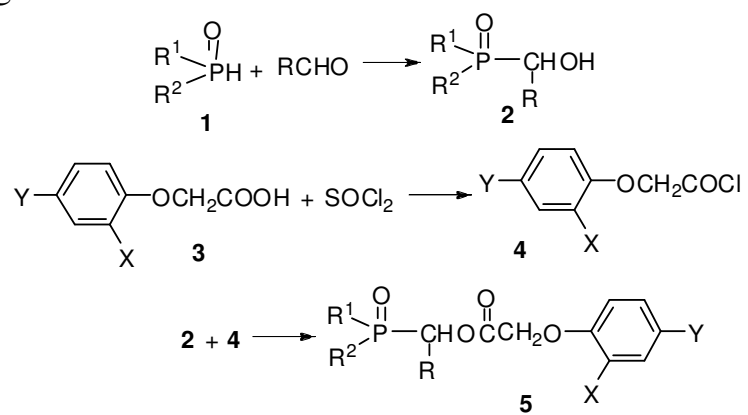

"X, Y = Cl, $\mathrm{NO}_{2}, \mathrm{R}=\mathrm{n}-\mathrm{Pr}, \mathrm{Ph}, \mathrm{m}-\mathrm{NO}_{2} \mathrm{Ph}, \mathrm{p}-\mathrm{NO}_{2} \mathrm{Ph}, \mathrm{R}^{1}=\mathrm{OCH}_{3}, \mathrm{CH}_{3}, \mathrm{R}^{2}=\mathrm{OCH}_{3}$ "

Scheme 1: Synthetic pathway of 1-(substituted phenoxyacetoxy) alkyl phosphonates and phosphinates

\section{General synthetic procedure for compound 5}

To a stirred and cooled solution of the appropriate $\alpha$-hydroxyalkyl phosphonate or phosphinate $(0.01 \mathrm{~mol})$ in $10 \mathrm{ml}$ chloroform and $0.8 \mathrm{ml}$ pyridine, substituted phenoxyacetyl chloride $(0.012 \mathrm{~mol})$ dissolved in $10 \mathrm{ml}$ chloroform was added slowly to keep the reaction temperature below $10^{\circ} \mathrm{C}$. The resultant mixture was stirred at an ambient temperature for 4-6 h, washed successively with $1 \% \mathrm{NaOH}$ $(3 \times 10 \mathrm{ml})$ and saturated $\mathrm{NaCl}(2 \times 10 \mathrm{ml})$. The organic phase was dried over anhydrous $\mathrm{Na}_{2} \mathrm{SO}_{4}$ and concentrated in vacuo. The residue was purified by column 
chromatography on silica gel using acetone+petroleum ether $(1+1)$ as an eluent to give 5a-n.

\section{Methods of bioassay}

\section{(a) Herbicidal activity test in Greenhouse}

Plastic pots were packed with sandy clay loam soil and water was added up to $3 \mathrm{~cm}$ in depth. Plant seeds were sown in the pots, and a diluted suspension of each compound containing acetone and Tween 80 was applied into the pots at $0.225 \mathrm{gm}^{-2}$. Five days later, the pre-emergence herbicidal activity against each plant was visually evaluated. The solution of the chemicals tested was applied to the foliage of plants grown at the 2-3 leaves stage with a sprayer at the rate of $0.225 \mathrm{~g} \mathrm{~m}^{-2}$ with a spelling volume of $100 \mathrm{ml} \mathrm{m}^{-2}$. Test plants were harvested 15 days after treatment. The fresh weights of aerial parts of each plant were measured. The post-emergence herbicidal activity against each plant was evaluated. Each experiment was replicated two times.

\section{(b) Determination of $I C_{50}$}

A compound with a certain concentration was dissolved in acetone and placed on a filter paper $(5.5 \mathrm{~cm}$ diameter) in Petri dishes $(9 \mathrm{~cm})$, and 10 cucumber seeds were placed on the filter paper after soaking in water for $6 \mathrm{~h}$. The Petri dishes with cucumber seeds were placed in a LRH-250-G lighting culture tank at $28^{\circ} \mathrm{C}$ for 3 days with $10 \mathrm{~h}$ of lighting and $14 \mathrm{~h}$ in the dark. After 6 days of cultivation, the inhibition percentage was calculated by the corresponding control using the length of the taproot or stem as an indicator. Three replications per concentration were performed. According to the average inhibition of cucumber root or stem at five concentrations for each compound, $\mathrm{IC}_{50}$ was estimated by regression analysis using the logarithm of concentration and a probit of corresponding inhibition percentage.

The wheat coleoptile test and Cucumber cotyledon test procedures were the same as described in the literature ${ }^{16}$.

\section{Results and Discussion}

\section{Chemistry}

Compound 1 is O,O-dimethyl phosphite or O-methyl methyl phosphite. O,ODimethyl phosphite was used directly as obtained commercially or prepared by the reaction of phosphorus trichloride and methanol. ${ }^{17}$ O-methyl methyl phosphite was synthesized in two-step sequence starting from phosphorus trichloride, methyl iodide and aluminium trichloride. ${ }^{10,11}$ Compound $\mathbf{2}$ was prepared by addition of compound 1 to the desired aldehyde using triethylamine as catalyst in yields of 53$86 \%$. $^{12,13}$ Compound $\mathbf{3}$ could be easily synthesized according to the literature 
J. Nepal Chem. Soc., Vol. 23, 2008/2009

procedures, ${ }^{1,14,15}$ starting from substituted phenol and 2-chloroacetic acid or 2bromoacetic ester in yields of $68-75 \%$.

The preparation of the title compounds involved the condensation of substituted phenoxyacetyl chloride $\mathbf{4}$ and $O, O$-dimethyl 1-hydroxylalkyl phosphonates or $O$-methyl 1-hydroxylalkyl methyl phosphinates 2 . The synthetic pathway is outlined in Scheme 1 and the structures of 5a-n are given in Table 1.

All new compounds were identified by ${ }^{1} \mathrm{H}$ NMR (Table 2), IR (Table 3) and elemental analysis (Table 1). In the ${ }^{1} \mathrm{H}$ NMR spectra of 5a-n: the protons in the P-C moieties and $\mathrm{P}-\mathrm{OCH}_{3}$ moiety display doublets, which are due to coupling with the phosphorus. All the main functional groups were characterized using IR spectra. A strong absorption near $1760 \mathrm{~cm}^{-1}$ was identified for the $\mathrm{C}=\mathrm{O}$. A sharp, weak band at $3050-3100 \mathrm{~cm}^{-1}$ accounted for the $\mathrm{C}-\mathrm{H}$ stretching of the benzene ring. A strong peak at around $1250 \mathrm{~cm}^{-1}$ accounted for $\mathrm{P}=\mathrm{O}$ in phosphonates, and at around $1180 \mathrm{~cm}^{-1}$ for $\mathrm{P}=\mathrm{O}$ in phosphinates. Two strong peaks at $1520 \mathrm{~cm}^{-1}$ and at $1350 \mathrm{~cm}^{-1}$ were the evidence for the $\mathrm{NO}_{2}$ stretching.

Table 1: Yield, melting point or refractive index and elemental analysis data of compounds (5a-n).

\begin{tabular}{|c|c|c|c|c|c|c|c|c|c|}
\hline \multirow[t]{2}{*}{ Compd. } & \multirow[t]{2}{*}{$\mathrm{R}^{1}$} & \multirow[t]{2}{*}{$\mathrm{R}$} & \multirow[t]{2}{*}{$X$} & \multirow[t]{2}{*}{$\mathrm{Y}$} & \multirow{2}{*}{$\begin{array}{c}\text { Yield }^{\mathrm{a}} \\
(\%)\end{array}$} & \multirow{2}{*}{$\begin{array}{l}\mathrm{Mp}\left({ }^{\circ} \mathrm{C}\right) \\
\mathrm{n}_{\mathrm{D}}^{20}\end{array}$} & \multicolumn{3}{|c|}{ Analysis Found (Calcd.) (\%) } \\
\hline & & & & & & & $\mathrm{C}$ & $\mathrm{H}$ & $\mathrm{N}$ \\
\hline $5 \mathbf{a}$ & $\mathrm{OCH}_{3}$ & $m-\mathrm{NO}_{2} \mathrm{Ph}$ & $\mathrm{Cl}$ & $\mathrm{Cl}$ & 52 & $92-93^{b}$ & 43.93(43.96) & $3.78(3.45)$ & $3.16(3.02)$ \\
\hline $5 b$ & $\mathrm{OCH}_{3}$ & $p-\mathrm{NO}_{2} \mathrm{Ph}$ & $\mathrm{Cl}$ & $\mathrm{Cl}$ & 54 & $126-127^{\mathrm{b}}$ & 43.74(43.96) & $3.82(3.45)$ & $3.18(3.02)$ \\
\hline $5 c$ & $\mathrm{OCH}_{3}$ & $m-\mathrm{NO}_{2} \mathrm{Ph}$ & $\mathrm{NO}_{2}$ & $\mathrm{H}$ & 51 & $86-87$ & $46.49(46.36)$ & $4.09(3.86)$ & $6.31(6.36)$ \\
\hline 5d & $\mathrm{OCH}_{3}$ & $p-\mathrm{NO}_{2} \mathrm{Ph}$ & $\mathrm{NO}_{2}$ & $\mathrm{H}$ & 55 & $162-163$ & $46.83(46.36)$ & $4.21(3.86)$ & $6.44(6.36)$ \\
\hline $5 e$ & $\mathrm{OCH}_{3}$ & $\mathrm{Ph}$ & $\mathrm{H}$ & $\mathrm{NO}_{2}$ & 61 & $157-158$ & $51.60(51.65)$ & $4.57(4.56)$ & $3.47(3.54)$ \\
\hline $\mathbf{5 f}$ & $\mathrm{OCH}_{3}$ & $m-\mathrm{NO}_{2} \mathrm{Ph}$ & $\mathrm{H}$ & $\mathrm{NO}_{2}$ & 52 & $93-94$ & $46.25(46.36)$ & $4.02(3.86)$ & $6.41(6.36)$ \\
\hline $5 \mathrm{~g}$ & $\mathrm{OCH}_{3}$ & $n-\operatorname{Pr}$ & $\mathrm{H}$ & $\mathrm{NO}_{2}$ & 66 & $33-34$ & $46.36(46.54)$ & $6.06(5.54)$ & $4.32(3.88)$ \\
\hline $5 h$ & $\mathrm{OCH}_{3}$ & $\mathrm{Ph}$ & $\mathrm{H}$ & $\mathrm{Cl}$ & 76 & $95-97$ & $53.55(53.07)$ & $4.22(4.72)$ & \\
\hline $5 i$ & $\mathrm{OCH}_{3}$ & $m-\mathrm{NO}_{2} \mathrm{Ph}$ & $\mathrm{H}$ & $\mathrm{Cl}$ & 80 & $80-84$ & $47.01(47.51)$ & $3.97(3.26)$ & $3.02(3.26)$ \\
\hline $5 \mathbf{j}$ & $\mathrm{CH}_{3}$ & $m-\mathrm{NO}_{2} \mathrm{Ph}$ & $\mathrm{Cl}$ & $\mathrm{Cl}$ & 82 & 1.5550 & $45.76(45.56)$ & $3.18(3.60)$ & $2.96(3.12)$ \\
\hline $5 \mathbf{k}$ & $\mathrm{CH}_{3}$ & $n-\operatorname{Pr}$ & $\mathrm{Cl}$ & $\mathrm{Cl}$ & 84 & $97-99$ & $45.40(45.55)$ & $5.10(5.19)$ & \\
\hline 51 & $\mathrm{CH}_{3}$ & $m-\mathrm{NO}_{2} \mathrm{Ph}$ & $\mathrm{NO}_{2}$ & $\mathrm{H}$ & 80 & 1.5403 & $47.90(48.12)$ & $4.13(4.04)$ & $6.55(6.60)$ \\
\hline $5 \mathrm{~m}$ & $\mathrm{CH}_{3}$ & $n-\operatorname{Pr}$ & $\mathrm{NO}_{2}$ & $\mathrm{H}$ & 68 & 1.5122 & $48.85(48.70)$ & $5.98(5.84)$ & $4.26(4.05)$ \\
\hline 50 & $\mathrm{CH}_{3}$ & $m-\mathrm{NO}_{2} \mathrm{Ph}$ & $\mathrm{Cl}$ & $\mathrm{NO}_{2}$ & 57 & $60-62$ & $44.51(44.50)$ & $3.65(3.52)$ & $6.22(6.10)$ \\
\hline
\end{tabular}


J. Nepal Chem. Soc., Vol. 23, 2008/2009

\section{Biological Assays}

The herbicidal activity of title compounds 5a-n was evaluated at a rate of $0.225 \mathrm{~g} \mathrm{~m}^{-2}$ in a set of experiments in greenhouse. They were tested for preemergence and post-emergence inhibitory effects against Echinochloa Crusgalli Beava (barngard grass), Digitaria Sanguinalis scop (ascendant crabgrass), Brassica napus L. (rape), Cirsium arvensis (thistle), and Cucumis sativa L. (cucumber). The results are listed in Table 4.

Table 2: ${ }^{1} \mathrm{H}$ NMR spectral properties of compounds (5a-n).

\begin{tabular}{|c|c|}
\hline Compd. & ${ }^{1} \mathrm{H}$ NMR $\left(\mathrm{CDCl}_{3} / \mathrm{TMS}\right), \delta$ \\
\hline $5 a^{a}$ & $\begin{array}{l}3.88\left(\mathrm{dd}, 6 \mathrm{H}, 2-\mathrm{OCH}_{3}\right), 5.09\left(\mathrm{~s}, 2 \mathrm{H} .-\mathrm{OCH}_{2}-\right), 5.67(\mathrm{~d}, 1 \mathrm{H},-\mathrm{CHP}-), \\
7.03-8.16\left(\mathrm{~m}, 7 \mathrm{H}, \mathrm{C}_{6} \mathrm{H}_{3}+\mathrm{C}_{6} \mathrm{H}_{4}\right)\end{array}$ \\
\hline $5 b^{a}$ & $\begin{array}{l}3.86\left(\mathrm{dd}, 6 \mathrm{H}, 2-\mathrm{OCH}_{3}\right), 5.01\left(\mathrm{~s}, 2 \mathrm{H},-\mathrm{OCH}_{2}-\right), 5.92(\mathrm{~d}, 1 \mathrm{H},-\mathrm{CHP}-), \\
7.00-8.02\left(\mathrm{~m}, 7 \mathrm{H}, \mathrm{C}_{6} \mathrm{H}_{3}+\mathrm{C}_{6} \mathrm{H}_{4}\right)\end{array}$ \\
\hline $5 c$ & $\begin{array}{l}\left.\text { 3.76-3.82 (dd, } 6 \mathrm{H}, 2-\mathrm{OCH}_{3}\right), 4.98\left(\mathrm{~s}, 2 \mathrm{H},-\mathrm{OCH}_{2}-\right), 6.36(\mathrm{~d}, 1 \mathrm{H},-\mathrm{CHP}-) \text {, } \\
7.02-8.30\left(\mathrm{~m}, 8 \mathrm{H}, \mathrm{C}_{6} \mathrm{H}_{4}+\mathrm{C}_{6} \mathrm{H}_{4}\right)\end{array}$ \\
\hline 5d & $\begin{array}{l}\left.\text { 3.92-3.95 (dd, } 6 \mathrm{H}, 2-\mathrm{OCH}_{3}\right), 4.87\left(\mathrm{~s}, 2 \mathrm{H} .-\mathrm{OCH}_{2}-\right), 6.34(\mathrm{~d}, 1 \mathrm{H},-\mathrm{CHP}-) \text {, } \\
\left.\text { 7.15-8.17 (m, } 8 \mathrm{H}, \mathrm{C}_{6} \mathrm{H}_{4}+\mathrm{C}_{6} \mathrm{H}_{4}\right)\end{array}$ \\
\hline $5 e$ & $\begin{array}{l}\left.\text { 3.58-3.66 (dd, } 6 \mathrm{H}, 2-\mathrm{OCH}_{3}\right), 4.82\left(\mathrm{~s}, 2 \mathrm{H},-\mathrm{OCH}_{2}-\right), 6.19(\mathrm{~d}, 1 \mathrm{H},-\mathrm{CHP}-), \\
\left.\text { 6.84-8.16(m, } 9 \mathrm{H}, \mathrm{C}_{6} \mathrm{H}_{4}+\mathrm{C}_{6} \mathrm{H}_{5}\right)\end{array}$ \\
\hline $\mathbf{5 f}$ & $\begin{array}{l}3.66-3.78\left(\mathrm{dd}, 6 \mathrm{H}, 2-\mathrm{OCH}_{3}\right), 4.88\left(\mathrm{~s}, 2 \mathrm{H},-\mathrm{OCH}_{2}-\right), 6.29(\mathrm{~d}, 1 \mathrm{H},-\mathrm{CHP}-) \\
6.90-8.22\left(\mathrm{~m}, 8 \mathrm{H}, \mathrm{C}_{6} \mathrm{H}_{4}+\mathrm{C}_{6} \mathrm{H}_{4}\right)\end{array}$ \\
\hline $5 g$ & $\begin{array}{l}0.92\left(\mathrm{t} .3 \mathrm{H},-\mathrm{CH}_{3}\right), 1.25-2.02\left(\mathrm{~m}, 4 \mathrm{H}, 2-\mathrm{CH}_{2}-\right), 3.70-3.84\left(\mathrm{dd}, 6 \mathrm{H}, 2-\mathrm{OCH}_{3}\right), \\
4.80\left(\mathrm{~s}, 2 \mathrm{H},-\mathrm{OCH}_{2}-\right), 5.22-5.46(\mathrm{~m}, 1 \mathrm{H},-\mathrm{CHP}-), 6.90-8.22\left(\mathrm{~m}, 4 \mathrm{H},-\mathrm{C}_{6} \mathrm{H}_{4}\right)\end{array}$ \\
\hline $5 \mathrm{~h}$ & $\begin{array}{l}3.62-3.71\left(\mathrm{dd}, 6 \mathrm{H}, 2-\mathrm{OCH}_{3}\right), 4.73\left(\mathrm{~s}, 2 \mathrm{H},-\mathrm{OCH}_{2}-\right), 6.25(\mathrm{~d}, 1 \mathrm{H},-\mathrm{CHP}-) \\
6.81-7.46\left(\mathrm{~m}, 9 \mathrm{H}, \mathrm{C}_{6} \mathrm{H}_{5}+\mathrm{C}_{6} \mathrm{H}_{4}\right)\end{array}$ \\
\hline $5 i$ & $\begin{array}{l}3.72-3.82\left(\mathrm{dd}, 6 \mathrm{H}, 2-\mathrm{OCH}_{3}\right), 4.79\left(\mathrm{~s}, 2 \mathrm{H},-\mathrm{OCH}_{2}-\right), 6.34(\mathrm{~d}, 1 \mathrm{H},-\mathrm{CHP}-), \\
6.81-8.29\left(\mathrm{~m}, 8 \mathrm{H}, \mathrm{C}_{6} \mathrm{H}_{4}+\mathrm{C}_{6} \mathrm{H}_{4}\right)\end{array}$ \\
\hline $5 \mathbf{j}$ & $\begin{array}{l}1.39-1.54\left(\text { dd. } 3 \mathrm{H},-\mathrm{CH}_{3}\right), 3.61-3.74\left(\mathrm{dd}, 3 \mathrm{H},-\mathrm{OCH}_{3}\right), 4.97\left(\mathrm{~s}, 2 \mathrm{H},-\mathrm{OCH}_{2}-\right) \text {, } \\
6.27-6.42(\mathrm{dd}, 1 \mathrm{H},-\mathrm{CHP}-), 6.82-8.28\left(\mathrm{~m}, 7 \mathrm{H}, \mathrm{C}_{6} \mathrm{H}_{4}+\mathrm{C}_{6} \mathrm{H}_{3}\right)\end{array}$ \\
\hline $5 k$ & $\begin{array}{l}0.90-0.97\left(\mathrm{t}, 3 \mathrm{H},-\mathrm{CH}_{3}\right), 1.34-1.41\left(\mathrm{dd}, 3 \mathrm{H},-\mathrm{CH}_{3}\right) \\
1.46-1.80\left(\mathrm{~m}, 4 \mathrm{H}, 2-\mathrm{CH}_{2^{-}}\right), 3.65-3.79\left(\mathrm{dd}, 3 \mathrm{H},-\mathrm{OCH}_{3}\right) \\
4.78\left(\mathrm{~s}, 2 \mathrm{H},-\mathrm{OCH}_{2}\right), 5.30-5.48(\mathrm{~m}, 1 \mathrm{H},-\mathrm{CHP}), 6.75-7.41\left(\mathrm{~m}, 3 \mathrm{H}, \mathrm{C}_{6} \mathrm{H}_{3}\right)\end{array}$ \\
\hline 51 & $\begin{array}{l}1.32-1.54\left(\text { dd. } 3 \mathrm{H},-\mathrm{CH}_{3}\right), 3.55-3.73\left(\mathrm{dd}, 3 \mathrm{H},-\mathrm{OCH}_{3}\right), 4.96\left(\mathrm{~s}, 2 \mathrm{H},-\mathrm{OCH}_{2}-\right) \text {, } \\
6.16-6.36(\mathrm{t}, 1 \mathrm{H},-\mathrm{CHP}-), 6.95-8.17\left(\mathrm{~m}, 8 \mathrm{H}, \mathrm{C}_{6} \mathrm{H}_{4}+\mathrm{C}_{6} \mathrm{H}_{4}\right)\end{array}$ \\
\hline $5 \mathrm{~m}$ & $\begin{array}{l}0.83-0.98\left(\mathrm{t}, 3 \mathrm{H},-\mathrm{CH}_{3}\right), 1.32-1.50\left(\mathrm{dd} .3 \mathrm{H},-\mathrm{CH}_{3}\right) \\
1.62-1.98\left(\mathrm{~m}, 4 \mathrm{H}, 2-\mathrm{CH}_{2}-\right), 3.57-3.77\left(\mathrm{dd}, 3 \mathrm{H},-\mathrm{OCH}_{3}\right) \\
4.81\left(\mathrm{~s}, 2 \mathrm{H},-\mathrm{OCH}_{2}-\right), 5.16-5.44(\mathrm{~m}, 1 \mathrm{H},-\mathrm{CHP}-), 6.94-7.86\left(\mathrm{~m}, 4 \mathrm{H}, \mathrm{C}_{6} \mathrm{H}_{4}\right)\end{array}$ \\
\hline $5 n$ & $\begin{array}{l}1.41-1.51\left(\mathrm{dd} .3 \mathrm{H},-\mathrm{CH}_{3}\right), 3.65-3.74\left(\mathrm{dd}, 3 \mathrm{H},-\mathrm{OCH}_{3}\right), 5.06\left(\mathrm{~s}, 2 \mathrm{H},-\mathrm{OCH}_{2}-\right) \text {, } \\
6.26-6.38(\mathrm{dd}, 1 \mathrm{H},-\mathrm{CHP}-), 6.94-8.35\left(\mathrm{~m}, 7 \mathrm{H}, \mathrm{C}_{6} \mathrm{H}_{4}+\mathrm{C}_{6} \mathrm{H}_{3}\right)\end{array}$ \\
\hline
\end{tabular}

As Table 4 indicates, there were remarkable differences among the herbicidal activity of the title compounds. Compounds 5a, 5b, 5h-5k showed much better activity than that of compounds $\mathbf{5 c - 5 g}, \mathbf{5 l - 5}$. In particular, compounds $\mathbf{5 a}, \mathbf{5 b}, \mathbf{5} \mathbf{j}$, 5k showed 100\% inhibitory effect against dicotyledon (Brassica napus L., Cirsium arvensis, Cucumis sativ L.) for post-emergence. Compounds $\mathbf{5 j}$, 5k also exhibited 83.3-100\% inhibitory effect against monocotyledon (Echinochloa Crusgalli Beava, Digitaria Sanguinalis scop) for both pre-emergence and post-emergence. 
J. Nepal Chem. Soc., Vol. 23, 2008/2009

Table 3: IR spectral properties of compounds $\mathbf{5 a}-\boldsymbol{n}\left(\mathrm{v} / \mathrm{cm}^{-1}\right)$.

\begin{tabular}{|c|c|c|c|c|c|c|c|c|c|}
\hline Compd. & $\mathrm{Ar}-\mathrm{H}$ & R-H & $\mathrm{C}=\mathrm{O}$ & $\mathrm{C}=\mathrm{C}$ & $\begin{array}{c}\mathrm{C}- \\
\mathrm{NO}_{2}\end{array}$ & $\mathrm{P}=\mathrm{O}$ & $\begin{array}{c}\text { C-O- } \\
\text { C }\end{array}$ & $\mathrm{P}-\mathrm{O}-\mathrm{C}$ & $\mathrm{P}-\mathrm{C}$ \\
\hline \multirow[t]{2}{*}{$5 a^{a}$} & 3028 & 2952 & 1774 & 1580 & 1533 & 1235 & 1170 & 1035 & 758 \\
\hline & & 2845 & & 1460 & 1352 & & & & \\
\hline \multirow[t]{2}{*}{$5 b^{a}$} & 3043 & 2958 & 1773 & 1578 & 1521 & 1257 & 1176 & 1056 & 751 \\
\hline & & 2858 & & 1472 & 1348 & & & & \\
\hline \multirow[t]{2}{*}{$5 c$} & 3046 & 2951 & 1787 & 1590 & 1528 & 1253 & 1178 & 1037 & 745 \\
\hline & & 2856 & & 1497 & 1350 & & & & \\
\hline \multirow[t]{2}{*}{$5 d$} & 3030 & 2950 & 1780 & 1580 & 1520 & 1250 & 1183 & 1055 & 749 \\
\hline & & 2849 & & 1454 & 1352 & & & & \\
\hline \multirow[t]{2}{*}{$5 e$} & 3072 & 2961 & 1770 & 1591 & 1507 & 1267 & 1173 & 1048 & 748 \\
\hline & & 2858 & & 1448 & 1346 & & & & \\
\hline \multirow[t]{2}{*}{$5 f$} & 3082 & 2955 & 1787 & 1592 & 1525 & 1269 & 1171 & 1058 & 752 \\
\hline & & 2852 & & 1439 & 1344 & & & & \\
\hline \multirow[t]{2}{*}{$5 g$} & 3084 & 2960 & 1767 & 1593 & 1516 & 1261 & 1178 & 1032 & 751 \\
\hline & & 2855 & & 1496 & 1344 & & & & \\
\hline \multirow[t]{2}{*}{$5 h$} & 3072 & 2956 & 1776 & 1596 & & 1256 & 1165 & 1029 & 752 \\
\hline & & 2855 & & 1492 & & & & & \\
\hline \multirow[t]{2}{*}{$5 i$} & 3020 & 2959 & 1769 & 1595 & 1534 & 1223 & 1172 & 1037 & 733 \\
\hline & & 2857 & & 1492 & 1354 & & & & \\
\hline \multirow[t]{2}{*}{$5 \mathbf{j}$} & 3061 & 2944 & 1758 & 1579 & 1542 & 1170 & 1102 & 1038 & 1300 \\
\hline & & 2872 & & 1474 & 1350 & & & & 889 \\
\hline \multirow[t]{2}{*}{$5 \mathbf{k}$} & 3061 & 2958 & 1764 & 1587 & & 1180 & 1108 & 1034 & 1307 \\
\hline & & 2873 & & 1488 & & & & & 876 \\
\hline \multirow[t]{2}{*}{51} & 3058 & 2941 & 1762 & 1579 & 1528 & 1169 & 1115 & 1031 & 1305 \\
\hline & & 2856 & & 1490 & 1341 & & & & 889 \\
\hline \multirow[t]{2}{*}{$5 \mathrm{~m}$} & 3084 & 2961 & 1764 & 1587 & 1525 & 1188 & 1112 & 1038 & 1303 \\
\hline & & 2876 & & 1487 & 1353 & & & & 875 \\
\hline \multirow[t]{2}{*}{$5 n$} & 3087 & 2954 & 1775 & 1585 & 1524 & 1171 & 1126 & 1032 & 1288 \\
\hline & 3043 & 2853 & & 1489 & 1341 & & & & 877 \\
\hline
\end{tabular}

Among all the compounds, compound 5j showed the highest activity, which exhibited $97.3-100 \%$ post-emergence inhibitory activity and $90.0-100 \%$ preemergence inhibition against all the test plants in greenhouse. Compound 50 also listed in Table 4 was employed to discuss herbicidal activity. It was obtained from our previous work. ${ }^{19}$ Based on the preliminary bioassays, title compounds were tested for $\mathrm{IC}_{50}$ values against the growth of Cucumis Sativa L. The results are listed in Table 5.

According to the $\mathrm{IC}_{50}$ value shown in Table 5, the $\mathrm{IC}_{50}$ results corresponded well to the results in Table 1 . Compounds $\mathbf{5 a}, \mathbf{5 b}, \mathbf{5 h}-\mathbf{5 k}$, for which the $\mathrm{IC}_{50}$ values were less than $11.2 \mu \mathrm{M}$, showed higher inhibitory activity, whereas compounds $\mathbf{5 c}$ 5g, 5l-5n, for which the $\mathrm{IC}_{50}$ values were more than $109 \mu \mathrm{M}$, exhibited low inhibitory activity against the growth of the root and stem of Cucumis sativa L. Compound 5a displayed the highest inhibitory activity against the growth of the root of Cucumis sativa L. $\left(\mathrm{IC}_{50}=0.00343 \mu \mathrm{M}\right)$ and compound $\mathbf{5 b}$ displayed the highest 
inhibitory activity against the growth of the stem of Cucumis sativa $L$. $\left(\mathrm{IC}_{50}=2.22\right.$ $\mu \mathrm{M})$.

From the data in Tables 4 and 5, we noticed that $\mathrm{NO}_{2}$ as $\mathrm{X}$ or $\mathrm{Y}$ on the benzene ring had greater influence on the herbicidal activity. Such herbicidal activity could be enhanced by introducing 2-Cl-4- $\mathrm{Cl}$ to the benzene ring, whereas the introduction of $\mathrm{X}$ or $\mathrm{Y}$ as $\mathrm{NO}_{2}$ resulted in a sharp decrease in their herbicidal activity towards test plants. As a typical example, the herbicidal activity of compounds 5a and 5j ( $\mathrm{X}$ and $\mathrm{Y}$ as 2-Cl-4-Cl, $\mathrm{R}^{1}$ as $\mathrm{OCH}_{3}$ or $\mathrm{CH}_{3}, \mathrm{R}$ as $m-\mathrm{NO}_{2} \mathrm{Ph}$ ) was much higher than that of compounds $\mathbf{5 c}$ and $\mathbf{5 l}\left(\mathrm{X}\right.$ and $\mathrm{Y}$ as $2-\mathrm{NO}_{2}-4-\mathrm{H}, \mathrm{R}^{1}$ as $\mathrm{OCH}_{3}$ or $\mathrm{CH}_{3}, \mathrm{R}$ as $m-\mathrm{NO}_{2} \mathrm{Ph}$ ). However, the introduction of a nitro group as $\mathrm{R}$ had a favorable effect on herbicidal activity when $\mathrm{X}$ or/and $\mathrm{Y}$ as $\mathrm{Cl}$, such as in compounds $\mathbf{5 a}, \mathbf{5 b}, \mathbf{5 i}$, and $\mathbf{5 j}$. The structure of $\mathrm{R}^{1}$ bounded to $\mathrm{P}$ had also great influence on the herbicidal activity. The herbicidal activity could be further enhanced by introducing $\mathrm{R}^{1}$ as $\mathrm{CH}_{3}$ to $\mathrm{P}$. For instance, the herbicidal activity of phosphinates $\mathbf{5 j}$ and $\mathbf{5 k}$ ( $\mathrm{X}$ and $\mathrm{Y}$ as $2-\mathrm{Cl}-4-\mathrm{Cl}, \mathrm{R}^{1}$ as $\mathrm{CH}_{3}, \mathrm{R}$ as $m-\mathrm{NO}_{2} \mathrm{Ph}$ or $n-\mathrm{Pr}$ ) was higher than that of phosphonates $\mathbf{5 a}$ and $\mathbf{5 o}\left(\mathrm{X}\right.$ and $\mathrm{Y}$ as $2-\mathrm{Cl}-4-\mathrm{Cl}, \mathrm{R}^{1}$ as $\mathrm{OCH}_{3}$, $\mathrm{R}$ as $m-\mathrm{NO}_{2} \mathrm{Ph}$ or $n$-Pr) against the test plants, especially on monocotyledon.

Table 4: The herbicidal activity of the title compounds $\left(0.225 \mathrm{~g} \mathrm{~m}^{-2} \text {, relative inhibition of growth \% }\right)^{a}$

\begin{tabular}{|c|c|c|c|c|c|c|c|c|c|c|}
\hline \multirow[t]{2}{*}{ Compd. } & \multicolumn{2}{|c|}{ Ech. } & \multicolumn{2}{|c|}{ Dig. } & \multicolumn{2}{|c|}{ Bra. } & \multicolumn{2}{|c|}{ Cir. } & \multicolumn{2}{|c|}{ Сис. } \\
\hline & Pre & Post & Pre & Post & Pre & Post & Pre & Post & Pre & Post \\
\hline $5 a$ & $\begin{array}{l}-52.9 \\
\end{array}$ & -28.0 & -47.5 & -26.2 & $\begin{array}{l}-57.8 \\
\end{array}$ & -100 & $\begin{array}{l}-100 \\
\end{array}$ & -100 & -96.4 & -100 \\
\hline $5 b$ & -41.1 & -44.0 & -53.9 & -35.2 & -100 & -100 & -100 & -100 & -99.0 & -100 \\
\hline $5 c$ & -2.3 & 0 & -6.7 & -0.3 & -0.9 & 0 & -6.7 & +5.2 & -0.3 & -20.0 \\
\hline 5d & -4.1 & -17.3 & 0 & 0 & -5.4 & -1.2 & -7.9 & +4.8 & -9.8 & -10.6 \\
\hline $5 e$ & -0.8 & 0 & 0 & +5.2 & +2.8 & -5.5 & +17.6 & +7.3 & +6.7 & -5.3 \\
\hline $5 f$ & +1.1 & +2.5 & -5.3 & -0.3 & +16.7 & +1.1 & +3.9 & +2.5 & +20.0 & -5.3 \\
\hline $5 \mathrm{~g}$ & -15.0 & -9.6 & -0.1 & -2.6 & +19.4 & -20.3 & +2.9 & +5.4 & +6.7 & -5.3 \\
\hline $5 \mathrm{~h}$ & -80.9 & -70.5 & -98.5 & -47.3 & -91.7 & -72.2 & / & I & I & / \\
\hline $5 i$ & -94.7 & -46.3 & -84.6 & -9.2 & -97.7 & -94.4 & I & I & I & I \\
\hline $5 \mathbf{j}$ & -90 & -97.3 & -100 & -100 & -100 & -100 & -90.9 & -100 & -100 & -100 \\
\hline $5 k$ & -90 & -94.6 & -83.3 & -100 & -100 & -100 & -54.5 & -100 & -100 & -100 \\
\hline 51 & +5 & -27.0 & 0 & 0 & -7.1 & -21.6 & 0 & 0 & 0 & 0 \\
\hline $5 \mathrm{~m}$ & -5 & -2.7 & 0 & 0 & +2.8 & 0 & -36.4 & I & I & I \\
\hline $5 n$ & +20 & 0 & 0 & -28.6 & +11.3 & +15.7 & -36.4 & -35.7 & I & +22.7 \\
\hline 50 & -70 & -52.5 & -75.0 & -55.0 & -99.5 & -100 & -98.9 & -100 & -99.0 & -100 \\
\hline
\end{tabular}

${ }^{a}$ Ech: Echinochloa Crusgalli Beava; Dig: Digitaria Sanguinalis scop; Bra: Brassica napus L.; Cir: Cirsium arvensis; Cuc: Cucumis sativ L.; pre: pre-emergence; post: post-emergence; -: inhibition on the growth of plants; +: stimulation on the growth of plants; /: As there was not enough sample for the test, the data were unavailable. 
J. Nepal Chem. Soc., Vol. 23, 2008/2009

Table 5: The $I_{50}$ of the title compounds against Cucumis sativa $L(\mu M)$.

\begin{tabular}{lcc}
\hline Compd. & $\begin{array}{c}\text { Inhibition on cucumber Root } \\
\left(\mathrm{IC}_{50}\right)\end{array}$ & $\begin{array}{c}\text { Inhibition on cucumber stem } \\
\left(\mathrm{IC}_{50}\right)\end{array}$ \\
\hline $\mathbf{5 a}$ & 0.00343 & 5.02 \\
$\mathbf{5 b}$ & 0.112 & 2.22 \\
$\mathbf{5 c}$ & $>11364$ & $>1818$ \\
$\mathbf{5 d}$ & 423 & 446 \\
$\mathbf{5 e}$ & 222 & 190 \\
$\mathbf{5 f}$ & 296 & 922 \\
$\mathbf{5 g}$ & 207 & 109 \\
$\mathbf{5 h}$ & 1.03 & 6.89 \\
$\mathbf{5 i}$ & 0.471 & 4.24 \\
$\mathbf{5 j}$ & 0.283 & 3.48 \\
$\mathbf{5 k}$ & 0.455 & 11.2 \\
$\mathbf{5 l}$ & 3193 & $>2781$ \\
$\mathbf{5 m}$ & 3052 & $>4200$ \\
$\mathbf{5 n}$ & 1160 & $>2379$ \\
\hline
\end{tabular}

Title compounds were tested for plant growth regulatory activity by wheat (Triticum aestivum L.) coleoptiles and cucumber (Cucumis satival L.) cotyledon test. ${ }^{18}$ The results are listed in Table 6 . As there were not enough samples for compounds $\mathbf{5 h}$ and $\mathbf{5 i}$, the data were not available.

As seen from Table 6 , compound $\mathbf{5 b}$ showed the highest inhibitory activity towards the growth of wheat coleoptile at $100 \mu \mathrm{g} \mathrm{ml}^{-1}$. However, compounds $\mathbf{5 a}, \mathbf{5 j}$ m exhibited high stimulating activity at $10 \mu \mathrm{g} \mathrm{ml}^{-1}$ towards the growth of wheat coleoptile, which was higher than that of the standard comparison reagent IAA. All of the phosphonates 5a-g showed no effect or weak effect on the growth of cucumber cotyledon root at $10 \mu \mathrm{g} \mathrm{ml}^{-1}$, whereas phosphinates $\mathbf{5 j}$ and $\mathbf{5 k}$ had $100 \%$ inhibitory effect on the growth of cucumber cotyledon root at $10 \mu \mathrm{g} \mathrm{ml}^{-1}$.

It was also found that the plant growth regulatory activity of phosphinates (e.g. 5j, 5k and 5l) was higher than its corresponding phosphonates (e.g. 5a, 5o and 5c) at $10 \mu \mathrm{g} \mathrm{ml}^{-1}$. The $\mathrm{NO}_{2}$ and $\mathrm{Cl}$ as $\mathrm{X}$ or/and $\mathrm{Y}$ in the benzene ring also had different influence on the plant growth regulatory activity. For example, the stimulating activity of compounds $\mathbf{5 a}$ and $\mathbf{5 j}$ (X and $\mathrm{Y}$ as $2-\mathrm{Cl}-4-\mathrm{Cl}, \mathrm{R}^{1}$ as $\mathrm{OCH}_{3}$ or $\mathrm{CH}_{3}, \mathrm{R}$ as $m-\mathrm{NO}_{2} \mathrm{Ph}$ ) was much higher than that of compounds $\mathbf{5 c}$ and $\mathbf{5 l}(\mathrm{X}$ and $\mathrm{Y}$ as $2-\mathrm{NO}_{2}-4-\mathrm{H}, \mathrm{R}^{1}$ as $\mathrm{OCH}_{3}$ or $\mathrm{CH}_{3}, \mathrm{R}$ as $m-\mathrm{NO}_{2} \mathrm{Ph}$ ) on the growth of wheat coleoptile at $10 \mu \mathrm{g} \mathrm{ml}^{-1}$. These observations show that the bioactivity of the title compounds highly depends upon $\mathrm{X}$ and $\mathrm{Y}$ on the benzene ring and the $\mathrm{R}^{1}$ bounded to $\mathrm{P}$. 
J. Nepal Chem. Soc., Vol. 23, 2008/2009

Table 6: The plant growth regulating activity of the title compounds (\%) ${ }^{a}$

\begin{tabular}{|c|c|c|c|}
\hline \multirow[t]{2}{*}{ Compd. } & \multicolumn{2}{|c|}{ Wheat coleoptile } & \multirow{2}{*}{$\begin{array}{l}\text { Cucumber cotyledon root } \\
\qquad 10 \mu \mathrm{g} \mathrm{ml}^{-1}\end{array}$} \\
\hline & $100 \mu \mathrm{g} \mathrm{ml}^{-1}$ & $10 \mu \mathrm{g} \mathrm{ml}^{-1}$ & \\
\hline $\mathbf{5 a}$ & -1.1 & +26.3 & 0 \\
\hline $5 \mathbf{b}$ & -26.3 & -5.3 & 0 \\
\hline 5c & +3.2 & -9.5 & 0 \\
\hline 5d & +10.5 & +5.3 & +4.3 \\
\hline $5 e$ & +11.6 & -13.7 & 0 \\
\hline $5 f$ & +5.3 & +17.9 & 0 \\
\hline $5 g$ & +7.4 & +15.8 & +4.3 \\
\hline $5 \mathbf{j}$ & +1.1 & +41.0 & -100 \\
\hline 5k & +12.5 & +29.7 & -100 \\
\hline 51 & +17.2 & +22.7 & -30.8 \\
\hline $5 \mathrm{~m}$ & +23.9 & +41.0 & -23.1 \\
\hline $5 n$ & -15.2 & -3.8 & +37.5 \\
\hline 50 & -25.4 & +4.4 & l \\
\hline ABA & -18.4 & & \\
\hline IAA & & +20.0 & \\
\hline
\end{tabular}

\section{Conclusions}

Above results indicate that there were remarkably different effects on bioactivity by introducing nitro group in $\mathrm{R}$ or/and as $\mathrm{X}$ or $\mathrm{Y}$ into the phosphonates and phosphinates. The introduction of a nitro group as $\mathrm{X}$ or $\mathrm{Y}$ resulted in low bioactivity, especially low herbicidal activity. However, the introduction of a nitro group in $\mathrm{R}$ had a favorable effect on bioactivity. Excellent herbicidal activity and plant growth regulatory activity were achieved in the compounds $\mathbf{5 a}, \mathbf{5 b}, \mathbf{5 j}$ with $\mathrm{NO}_{2}$ in $\mathrm{R}, \mathrm{X}$ and $\mathrm{Y}$ as 2-Cl-4-Cl and $\mathrm{R}^{1}$ as $\mathrm{OCH}_{3}$ or $\mathrm{CH}_{3}$. So we can conclude that the introduction of a nitro group as $\mathrm{X}$ or $\mathrm{Y}$ is not beneficial to bioactivity of the title compounds. The satisfactory herbicidal activity and plant growth regulatory activity of the title compounds could be achieved by introducing a nitro group in $\mathrm{R}$ with a reasonable combination of $\mathrm{X}, \mathrm{Y}$ and $\mathrm{R}^{1}$. These results provided some interesting hints for further study of structure modification and structure-activity relationship of these compounds.

\section{Acknowledgements}

We gratefully acknowledge financial support of this work by the National Basic Research Program of China (2003CB114400) and the National Natural 
J. Nepal Chem. Soc., Vol. 23, 2008/2009

Science Foundation of China (20372023). We would like to express our appreciation to Professor Heiner Eckstein for his help in revising the manuscript.

\section{References}

1. M. A. Hamid, E. S. Ramadan, M. Hagar and E. E. Ashry, Synth. Commun., 2004, 34, 377.

2. S.W. Park and B. I. Lee, US Pat.51, 1992,39,564.

3. N. Shindo, JP Pat. 62,1987,123,102.

4. R. Kluger and C. D. Pike, J. Ame.r Chem. Soc., 1977, 99, 4504.

5. A. C. Baillie, K. Wright, B. J. Wright and C. G. Earnshaw, Pestic. Biochem. Physiol., 1988, 30, 103.

6. J. Wang, H.W. He and Z. J. Liu, Chinese Chem. Lett., 1997, 8, 943.

7. H. W. He and Z. J. Liu, Chinese J. Org. Chem., 2001, 21, 878.

8. H. W. He, T. Wang and J. L. Yuan, J. Organometallic Chem., 2005, 690, 2608.

9. T. Chen, P. Shen, Y. J. Li and H. W. He, Phosphorus, Sulfur and Silicon, 2006, 181, 2135.

10. S. Miroslaw, Synthesis, 1977, 7, 450.

11. K. A. Petrov, N. K. Blizynyuk, Y. V. Studnev and A. F. Kolomiets, Zhur. Obschch. Khim., 1961, 31, 179.

12. T. Agawa, T. Kubo and Y. Ohshiro, Synthesis, 1971, 1, 27.

13. M. Q. Li, H. W. He, W. B. Chen and Z. J. Liu, Journal of Central China Normal University (Nat. Sci.), 2000, 34,179.

14. E. S. Ibrahim, S. M. Rida, Y. A. Beltagy and M. M. Abdel-Khalek, Pharmazie, 1974, 29,581.

15. J. L. Brayer, L. Taliani and J. Tessier, EP Pat. 376,819 (1990).

16. H. W. He, L. P. Meng, L. M. Hu and Z. J. Liu, Chinese Journal of Pesticide Science, 2002, 4, 14.

17. H. McCombie and B. C. Saunders, J. Chem. Soc., 1945, 2, 380.

18. T. Wang and H. W. He, Phosphorus Sulfur and Silicon, 2004, 179, 2081.

19. J. Wang, X. F. Liu, S. Q. Wang, L. S. Wang and H. W. He, Chemical

Research and Application, 1998, 10, 182. 